\title{
MODELOS ESPACIALES DE ALGUNAS \\ POBLACIONES DE COLEÓPTEROS \\ TERRESTRES EN DOS ECOSISTEMAS \\ DEL BAJO GUADALQUIVIR
}

(S. W. España)

A. de los Santos ${ }^{1}$, C. Montes ${ }^{1}$ y L. Ramírez-Díaz ${ }^{2}$.

\section{SUMMARY}

The spatial distribution in some soil surface coleoptera populations from two typical mediterranean ecosystems: grassland and pine woodland; during an annual cycle and, by the use of different agregation indexes is analysed.

Generally, these studied populations show spatial patterns in «clumps» whith different contagious levels and a marked seasonal distribution.

In relation to the structural characteristics of these populations and their ecological requeriments the detected patterns are explained.

For its application the the analysis and interpretation of the density activity data, the efficiency of the various types of used agregations indexes is evaluated.

1.-Departamento de Zoología, Universidad de Murcia.

2.-Departamento de Ecología, Universidad de Murcia. 


\section{RESUMEN}

Se analiza durante un ciclo anual, y mediante el empleo de diferentes índices de agregación, la distribución espacial de algunas poblaciónes de coleópteros terrestres de la superficie del suelo en un pastizal y un pinar típicos mediterráneos.

De una forma general, las poblaciones estudiadas presentan modelos espaciales en «agregados», con diferentes niveles de contagio y una marcada distribución estacional.

Se interpretan los modelos detectados en relación con las características estructurales de las poblaciones y sus requerimientos ecológicos.

Se evalúa la eficiencia de los distintos tipos de índices de agregación, en relación con su aplicación al análisis e interpretación de los datos de densidad en actividad.

\section{INTRODUCCIÓN}

La distribución espacial de una población o la descripción del modelo de distribución de los individuos en el espacio que ocupan, es uno de los aspectos de mayor relevancia en ecología descriptiva (Greig-Smith, 1964).

El pattern, modelo o distribución espacial, se considera una propiedad fundamental de los sistemas vivos y su estudio constituye un tema de gran importancia en multitud de trabajos, incidiendo en la eficiencia del programa de muestreo y en el análisis e interpretación de los datos (Rojas, 1964).

El estudio del modelo espacial, tiene como objetivo fundamental el análisis metodológico que conduce a la interpretación ecológica del comportamiento de una población. En una etapa inicial cuyos resultados permiten una mejor comprensión del problema, así como la formulación de nuevas hipótesis para investigaciones posteriores.

La distribución espacial de poblaciones de coleópteros terrestres de la superficie del suelo, a partir de datos obtenidos por trampas de caída 
(«pit-fall traps») no ha sido abordado con profundidad. Tan sólo Grum (1973) y Den Boer (1979) analizan el modelo espacial de algunas poblaciones de coleópteros en relación con la utilización del hábitat.

Las técnicas de empleo de las trampas de caída constituyen actualmente un campo de trabajo de enorme interés y de amplia difusión en estudios de ecología descriptiva y funcional para poblaciones de artrópodos terrestres de la superficie del suelo (Greenslade, 1964; Duffey, 1962; Mitchell, 1963; Hayes, 1970; Obrtel, 1971; Gist y Grossley, 1973; Joosse, 1975; Luff, 1979; Baars, 1979; Grum, 1980; Price y Shepard, 1980).

Este estudio pretende detectar e interpretar diferentes modelos de distribución espacial de algunas poblaciones de coleópteros terrestres en dos ecosistemas característicos del área del Bajo Guadalquivir, así como, analizar su incidencia en el proceso de obtención y análisis de los datos.

\section{MATERIAL Y MÉTODO}

El trabajo se llevó a cabo en los Pinares de Almensilla, en la provincia de Sevilla, entre los términos municipales de Almensilla, Aznalcázar y Puebla del Río. El sustrato sólido lo constituyen cantos y arenas pliocenocontinentales, con topografía en lomas moderadamente recortadas por la erosión. El suelo, acidificable, y de potencial agrícola mediocre, está formado por arenas descalcificadas con horizontes de textura más fina que alternan con capas de gravas cementadas e impermeables.

La vegetación potencial de alcornoque y encinas ha sido sustituida por plantaciones de Pinus pinea en gran parte de su superficie. El sotobosque está formado por un matorral acidófilo térmico, existiendo amplias zonas de pastizales de terófitos efímeros característicos de arenales oligotróficos (González Bernáldez, 1978).

La toma de datos se realizó en dos ecosistemas diferentes en cuanto a sus características ambientales y de intervención humana. Por una parte, se estudió un sector constituido por un mosaico de pastizales, determinados por la acción combinada de factores tales como pendiente, microtopografía, textura, profundidad de la capa freática e intensidad 
de pastoreo. Por otra, se incluyó un sector de pinar, con sotobosque de jarales, relativamente bien conservado.

Para más detalle sobre las características del medio físico natural de la zona de estudio ver de los Santos (1983).

La técnica de captura consistió en la utilización de trampas de caída dispuestas en una trama reticular, de tal manera que quedaran incluidos la mayoría de los microhábitats que constituyen los ecosistemas estudiados.

Se empleó un nuevo diseño de trampa de caída en embudo compartimentado (de los Santos y otros, 1983) que unifica las ventajas de la amplia variedad de modelos existentes. La colocación de las trampas se realizó teniendo en cuenta los diferentes factores que intervienen en la eficiencia de la captura. (Luff, 1975; Adis, 1979).

Las trampas situadas en el pinar y en el pastizal estaban constituidas por 30 y 40 trampas respectivamente, en función de la superficie de estudio y dispuestas en hileras paralelas, separadas entre sí 5 metros ( $15 \times 100$ m., en el pinar y $25 \times 100 \mathrm{~m}$., en el pastizal).

El período de estudio comprendió desde Agosto de 1981 a Agosto de 1982. Las trampas fueron vaciadas en periodos aproximados de una semana. Los individuos fueron contados sobre el terreno y devueltos al medio, salvo una pequeña muestra seleccionada que se transportaba al laboratorio para el estudio de los ciclos de vida.

Los métodos estadísticos empleados para el análisis de los modelos espaciales de las poblaciones abarcaron desde el ajuste a distribuciones de probabilidad hasta el empleo de diferentes índices de agregación (Elliot, 1977; Southwood, 1978). Los índices de agregación escogidos se han utilizado tanto para el análisis del total de la captura como para el estudio del modelo espacial estacional. 
Varianza relativa

$$
\mathrm{S}^{2} / \overline{\mathrm{x}}
$$

Andreawartha y Birch, 1954.

Chi-cuadrado

$$
\mathrm{S}^{2}(\mathrm{n}-1) / \overline{\mathrm{x}} \quad \text { Debauche, } 1958 .
$$

Lexis «I» $\mathrm{L}$

Elliot 1977.

Morisita «I» $\delta \quad \mathrm{n}\left(\sum \mathrm{x}^{2}\right)-\mathrm{x} /\left(\sum \mathrm{x}\right) \stackrel{2}{-}\left(\sum \mathrm{x}\right) \quad$ Morisita, 1959.

Coeficiente de Charlier $\quad 100\left(\sqrt{\mathrm{S}^{2}-\overline{\mathrm{x}} / \mathrm{x}}\right) \quad$ Elliot 1977.

Green $« \mathrm{I} »_{\mathrm{G}} \quad\left(\mathrm{S}^{2} / \overline{\mathrm{X}}\right)-1 / \sum \mathrm{x}-1 \quad$ Green, 1966.

Ley Potencia Taylor $« b » \quad S^{2}=a \cdot \bar{x}^{b} \quad$ Taylor, 1961.

Binomial Negativa «K» $\overline{\mathrm{x}}^{2} / \mathrm{s}^{2}-\overline{\mathrm{x}} \quad$ Southwood, 1978.

Lefkovitch «I» $\alpha \quad(1 / 45) \tan ^{-1}\left(\mathrm{~S}^{2} / \sigma^{2}\right)-1 \quad$ Lefkovitch, 1966.

«Mean crowding» $\underset{* \mathrm{X} »}{*} \overline{\mathrm{X}}+\left(\mathrm{S}^{2} / \overline{\mathrm{X}}\right)-1 \quad$ LLoyd, 1967.

«Pahiness

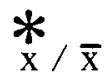

Lloyd, 1967.

Iwao $\langle\beta »$

$\stackrel{*}{*}=\alpha+\beta \overline{\mathrm{X}} \quad$ Iwao, 1968 .

\section{RESULTADOS}

En total se recogieron 16.610 individuos pertenecientes a 134 especies de 14 familias en el pastizal, y 694 individuos pertenecientes a 29 especies de 6 familias en el pinar (de los Santos, 1983).

Para el estudio de los modelos espaciales se consideraron 33 especies en el pastizal y 5 en el pinar, -en función de los valores de frecuencia y dominancia de las poblaciones-, pertenecientes a las familias Carabidae, Tenebrionidae, Curculionidae, Dermestidae, Scymaenidae, Histeridae, Silphidae, Scarabaeidae y Meloidae. 
En las figuras 1 y 2 se representan los valores de 12 índices de ajuste y agregación para el total de las capturas anuales de 38 poblaciones de coleópteros terrestres en los ecosistemas estudiados.

El ajuste de las frecuencias observadas al modelo de Poisson se realizó mediante la razón $S^{2} / \bar{x}$ contrastada mediante referencia a una distribución chi-cuadrado (Debauche, 1958; Greig-Smith, 1964). Se observa que la captura total anual en la trama de trampas presenta una razón $\mathrm{S}^{2} / \overline{\mathrm{X}}$ muy elevada (distribución contagiosa) y significativamente distinta a 1 , en los dos ecosistemas (Tablas 1 y 2). Tan sólo Hadrocarabus lucitanicus presenta, en el pinar, una distribución que se ajusta significativamente a una serie de Poisson, probablemente debido a su baja densidad (Elliot, 1977).

En una primera aproximación se puede utilizar la varianza relativa $\left(\mathrm{S}^{2} / \overline{\mathrm{x}}\right)$ para evaluar el grado de agregación de una población (Andrewartha y Birch, 1954). En las tablas 1 y 2 se observa que todas las poblaciones poseen valores elevados para este índice $\left(S^{2} / \bar{x}>1,67\right)$. Sin embargo, la utilización de la varianza relativa para determinar el nivel de agregación de una población presenta graves inconvenientes, ya que depende directamente de su abundancia (Levecue, 1972). Así, las especies dominantes, tanto del pastizal como del pinar (p.e.: Pimelia costata, Tentyria platyceps, Pterostichus globosus ebenus) poseen los valores más altos, mientras que las especies accesorias (p.e.: Licinus punctatulus granulatus, Platynus dorsalis) los valores son tan bajos que casi no son significativamente distintos a 1 .

Otros índices empleados y basados en el cociente $S^{2} / \bar{x}$ son el de Lexis « $\mathrm{I}_{\mathrm{L}}$ » y el de Lefkovitch «I $\alpha$ ». Estos índices ponen de manifiesto distribuciones contagiosas para todas las poblaciones estudiadas (Tablas 1 y 2). De igual forma, se ven afectados por las mismas consideraciones formuladas para la varianza relativa.

El coeficiente de Charlier $« \mathrm{C}_{\mathrm{C}} »$ es independiente del número total de individuos capturados y del valor medio de su abundancia, pero no del tamaño de la muestra. En este estudio se han utilizado el mismo número de unidades de muestreo para estimar la densidad de cada población. Por tanto, los valores obtenidos del índice $\left\langle\mathrm{C}_{C}\right\rangle$ pueden ser considerados como buenos indicadores del nivel de agregación de las poblaciones. Según este índice, las especies Licinus punctatulus granula- 


$\begin{array}{llllllllllll}(1) & (2) & (3) & (4) & (5) & (6) & (7) & (8) & (9) & (10) & (11) & (12) \\ s^{2} / \bar{x} & X^{2} & I_{L} & I_{\alpha} & C_{C} & I_{G} & b & k & I_{f} & * & * \\ x & \end{array}$

\section{CARABIDAE}

Pterostichus globosus ebenus quens Brachinus bellicosus Duf.

Brachinus sclopeta F.

Calosoma maderae $F$.

Bembidion varium ol.

Amara aenea Deg.

orthomus sp.

Hadrocarabus lucitanicus $F$.

Platynus dorsalis Pont.

Licinus punctatulus granulatus Dej.

\section{TENEBRIONIDAE}

Pimelia costata Walti.

Tentyria platyceps Stev.

Micrositus ulyssiponensis Germ.

Erodius goryi obtusus Alld.

sepidium bidentatum sol.

Scaurus punctatus $F$.

Grabrasida parallela Sol.

Litoborus planicollis walt.

CURCULIONIDAE

Brachycerus barbarus L.

Thylacites latithorax obr.

Rhytidoderes plicatus 0 I

Rhytirhinus sp.

Anysorhynchus sp.

Brachycerus callosu's sch.

DERMESTIDAE

Dermestes frischi Kug.

SCYDMAENIDAE

Mastigus palpalis Latr.

HISTERIDAE

Paromalus flavicornis Herbs. Saprinus semistriatus ser.

SILPHIDAE

Silpha striola Men.

SCARABAEIDAE

Trox perlatus hispanicus waltl.

Thyphoeus momus $\mathrm{E}$.

MELOIDAE

Meloe procarabaeus

\begin{tabular}{|c|c|c|c|c|c|c|c|c|c|c|c|}
\hline 20,88 & $814,60 *$ & 4,57 & 0,94 & 65,19 & 0,01 & 1,47 & 2,35 & 1,41 & 66,70 & 1,42 & 1,79 \\
\hline 49,15 & $2.402,90 *$ & 7,01 & 0,97 & 169,45 & 0,07 & 1,87 & 0,34 & 4,27 & 66,09 & 3,94 & 8,67 \\
\hline 76,88 & $2.998,30 \cdots$ & 8,77 & 0,98 & 319,13 & 0,26 & 2,04 & 0,10 & 10,96 & 83,33 & 11,18 & 23,54 \\
\hline 8,95 & $349,20 \bullet$ & 2,99 & 0,86 & 127,73 & 0,04 & 1,49 & 0,61 & 1,71 & 12,84 & 1,69 & 2,50 \\
\hline 6,32 & $243,19 \bullet \bullet$ & 2,49 & 0,80 & 144,67 & 0,05 & 1,67 & 0,48 & 3,06 & 7,73 & 3,09 & 4,72 \\
\hline 2,71 & $105,60 \bullet \bullet$ & 2,60 & 0,65 & 88,63 & 0,02 & 1,51 & 1,27 & 1,78 & 3,88 & 1,79 & 4,21 \\
\hline 3,59 & $140,00 *$ & 1,89 & 0,65 & 138,55 & 0,05 & 1,34 & 0,52 & 2,91 & 3,94 & 2,92 & 3,70 \\
\hline 5,84 & $227,80 \bullet$ & 2,41 & 0,78 & 228,69 & 0,13 & - & 0,29 & 6,25 & 5,76 & 6,23 & - \\
\hline 3,17 & $123,60 \bullet$ & 1,78 & $0,7.6$ & 159,76 & 0,07 & - & 0,39 & 3,57 & 3.03 & 3,56 & - \\
\hline 11,57 & $416,47 \bullet$ & 3,40 & 0,89 & 361,04 & 0,36 & - & 0,08 & 15,26 & 11,38 & 14,98 & - \\
\hline 8,30 & $298,85 * \bullet$ & 2,88 & 0,85 & 387,50 & 0,43 & - & 0,07 & 17,78 & 7,79 & 16,83 & - \\
\hline 53,07 & $2.069,70 \bullet$ & 7,29 & 0,98 & 72,62 & 0.01 & 1,73 & 1,86 & 1,51 & 151,75 & 1,53 & 2,17 \\
\hline 38,45 & $1.4,99,80 *$ & 6,20 & 0,96 & 76,47 & 0,01 & 1,65 & 1,71 & 1,57 & 101,50 & 1,58 & 1,94 \\
\hline 24,19 & $943,50 *$ & 4,90 & 0,95 & 78,49 & 0,01 & 1,69 & 1,62 & 1,57 & 60,84 & 2,62 & 2,55 \\
\hline 99,90 & $3.896,10 * *$ & 9,99 & 0,98 & 188,79 & 0,10 & 1,45 & 0,28 & 4,48 & 126,85 & 4,57 & 4,27 \\
\hline 10,19 & $397,57 \bullet$ & 3,19 & 0,87 & 111,09 & 0,03 & 1,56 & 0,81 & 2,21 & 16,64 & 2,23 & 5,21 \\
\hline 10,48 & $409,00 \bullet$ & 3,24 & 0,88 & 119,00 & 0,04 & 2,34 & 0,71 & 2,38 & 16,19 & 2,42 & 6,25 \\
\hline 4,61 & $179,99 \bullet *$ & 2,15 & 0,73 & 268,80 & 0,19 & 1,46 & 0,14 & 8,42 & 4,12 & 8,25 & 4,53 \\
\hline 2,73 & $92,57 \ldots$ & 1,54 & 0,49 & 169,90 & 0,08 & - & 0,35 & 3,97 & 2,85 & 3,89 & - \\
\hline 4,96 & $178,50 *$ & 2,23 & 0.75 & 94,49 & 0,02 & 1,40 & 1,12 & 2,03 & 8,39 & 1,99 & 2,37 \\
\hline 17,85 & $383,50 \bullet$ & 3,01 & 0,90 & 85,38 & 0,03 & 1,48 & 0,77 & 2,41 & 28,99 & 2,40 & 2,13 \\
\hline 11,28 & 440,10 & 3,36 & 0,89 & 174,50 & 0,08 & 2,39 & 0,33 & 4,00 & 13,66 & 4,05 & 8,45 \\
\hline 3,04 & $109,60 \bullet$ & 1,75 & 0,60 & 294,50 & 0,11 & - & 0,26 & 5,16 & 2,58 & 4,88 & - \\
\hline 1,79 & $69,96 *$ & 1,34 & 0,35 & 156,30 & 0,07 & - & 0.41 & 3.59 & 1,12 & 3,45 & - \\
\hline 2,76 & $68,66 *$ & 1,33 & 0,34 & 100,70 & 0,03 & - & 0,98 & 2,02 & 1,51 & 2,01 & - \\
\hline 76,05 & $2.887,90 *$ & 8,61 & 0,98 & 168,43 & 0,07 & 1,99 & 0.35 & 3,89 & 98,90 & 3,84 & 8,25 \\
\hline 62,17 & $2,424,70 \bullet$ & 7,88 & 0,98 & 305,60 & 0,23 & $2 ; 03$ & 0,11 & 9,94 & 67,76 & 10,30 & 17,66 \\
\hline 24,61 & $886,08 \bullet \bullet$ & 4,96 & 0,95 & 176,33 & 0,08 & 1,55 & 0,32 & 4,36 & 31,20 & 4,11 & 5,23 \\
\hline 7,66 & $298,90 *$ & 2.77 & 0,84 & 199,60 & 0,10 & - & 0.25 & 4,93 & 8,34 & 4,98 & - \\
\hline 5,95 & $232,26 \bullet \bullet$ & 2,44 & 0,79 & 207,50 & $0,1 I$ & 1,51 & 0,23 & 5,29 & 6,11 & 5,31 & 7,96 \\
\hline 2,41 & $93,99 \cdot$ & 1,55 & 0,50 & 106,20 & 0,03 & 1,44 & 0,89 & 2,12 & 2,66 & 2,13 & 1,78 \\
\hline 2,12 & $78,60 \cdots$ & 1,45 & 0,33 & 142,70 & 0,06 & - & 0,49 & 3,58 & 1,68 & 3,44 & - \\
\hline 1,76 & 63,53 . & 1,33 & 0,34 & 129,07 & 0,05 & - & 0,60 & 2,94 & 1,22 & 2,78 & - \\
\hline
\end{tabular}

- $P<0,01 ; \quad * P<0,005$

( 1 ) Varianza relativa; ( 2 ) Chi-cuadrado; ( 3 ) Indice de Lexis; ( 4 ) Indice de Lefkovitch; ( 5 ) coeficiente de charlier; ( 6 ) Indice de Green;

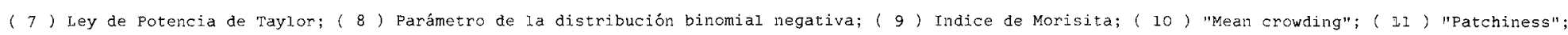
(12) Indice de Iwao.

- = Los datos obtenidos en las muestreos no permitieron el cálculo de los respectivos índices por la propia estructura de los mismos. 
Tabla 2 .- Valores de 12 indices de agregación para 5 poblaciones de coleópteros terrestres del pinar.

\begin{tabular}{|c|c|c|c|c|c|c|c|c|c|c|c|c|}
\hline & $\begin{array}{c}(1) \\
s^{2} / \bar{x}\end{array}$ & $\begin{array}{l}(2) \\
x^{2}\end{array}$ & $\left(\begin{array}{l}3 \\
I_{\mathbb{L}}\end{array}\right.$ & $\left(\begin{array}{l}4 \\
I_{\alpha}\end{array}\right.$ & $\left(\begin{array}{l}5 \\
C_{C}\end{array}\right.$ & $\begin{array}{l}(6) \\
I_{G}\end{array}$ & $\left(\begin{array}{l}7) \\
b\end{array}\right.$ & $(8)$ & $(9)$ & $\begin{array}{l}(10) \\
\underset{x}{*}\end{array}$ & $\begin{array}{l}(11) \\
\underset{\mathrm{*}}{*} / \overline{\mathrm{x}}\end{array}$ & $\begin{array}{l}(12) \\
\beta\end{array}$ \\
\hline TENEBRIONIDAE & & & & & & & & & & & & \\
\hline Pimelia costata Waltl. & 14,56 & $412,93 *$ & 3,77 & 0,91 & 102,00 & 0,04 & 1,55 & 0,96 & 1,84 & 25,99 & 2,04 & 3,05 \\
\hline Grabrasida parallela sol. & 8,79 & $254,85 *$ & 2,96 & 0,86 & 181,00 & 0,15 & 1,87 & 0,31 & 7,82 & 17,58 & 7,43 & 10,67 \\
\hline $\begin{array}{l}\text { Tentyria platyceps stev. } \\
\text { SCARABAEIDAE }\end{array}$ & 1,67 & 48,66 & 1,29 & 0,31 & 105,40 & 0,04 & - & 0,90 & 2,16 & 1,27 & 2,11 & - \\
\hline $\begin{array}{l}\text { Trox perlatus hispanicus Waltl. } \\
\text { CARABIDAE }\end{array}$ & 1,97 & $57,19 *$ & 1,40 & 0,40 & 59,20 & 0,02 & - & 1,16 & 1,97 & 5,18 & 1,87 & - \\
\hline Hadrocarabus lucitanicus F. & 0,71 & 20,20 & - & - & - & - & - & - & - & - & - & - \\
\hline
\end{tabular}

\section{$* \mathrm{P}<0,05 ; \quad * \mathrm{P}<0,005$}

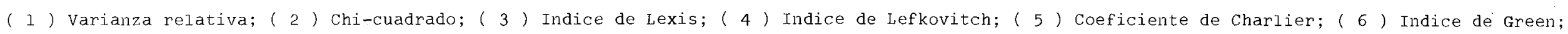

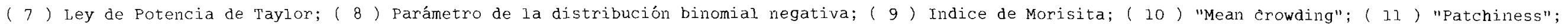
(12) Indice de Iwao.

- = Los datos obtenidos en los muestreos no permitieron el cálculo de los respectivos indices por la propia estructura de los mismos. 

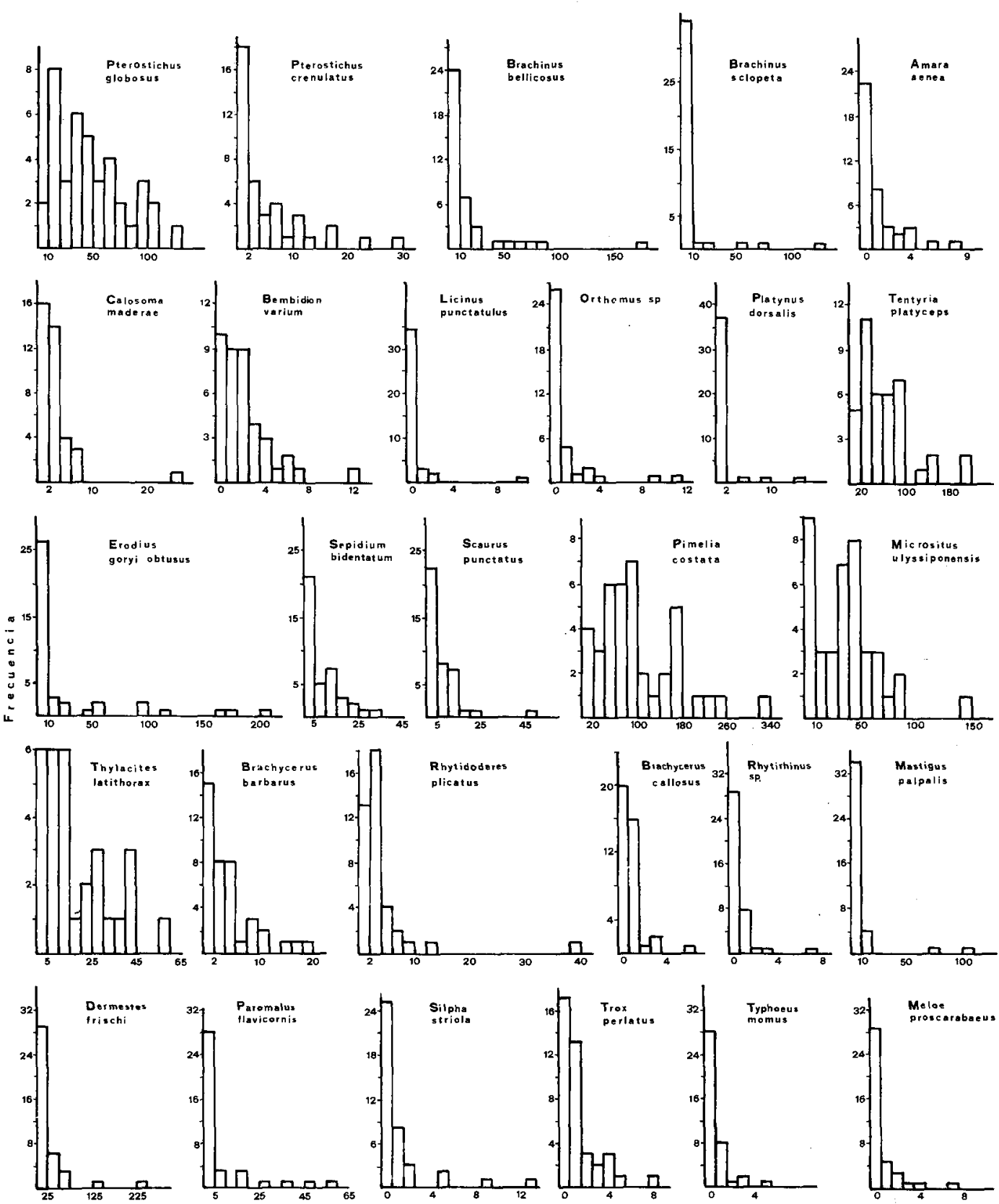

Figura 1. - Histogramas de frecuencias para el total de las capturas de 28 poblaciones de coleópteros del pastizal 

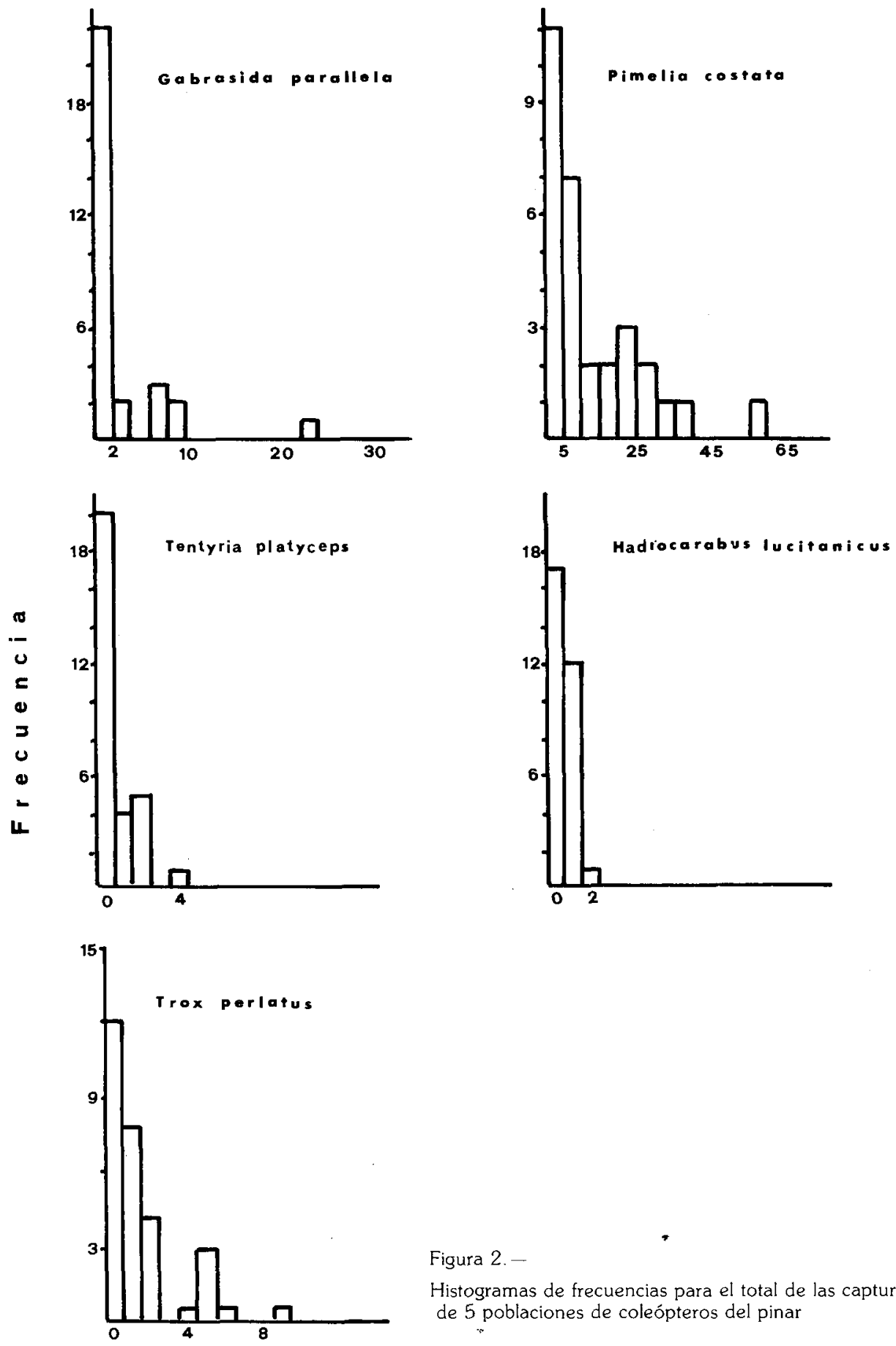

Figura 2.-

Histogramas de frecuencias para el total de las capturas de 5 poblaciones de coleópteros del pinar

Número de individuos 
tus, Platynus dorsalis (Fam. Carabidae), Dermestes frischi (Fam. Dermestidae), Silpha striola (Fam. Silphidae), Erodius goryi obtusus (Fam. Tenebrionidae) presentan valores de agregación muy elevados en el pastizal; mientras que en pinar, tan sólo Grabrasida paralela (Fam. Tenebrionidae) posee valores altos de agregación.

El resto de las poblaciones muestran una reducción gradual del nivel de agregación, apareciendo poblaciones con niveles medios (p.e.: Pterostichus crenulatus, Amara aenea, Scaurus punctatus) y bajos (Pterostichus globosus ebenus, Pimelia costata).

$\mathrm{El}$ índice de Green $" \mathrm{I}_{\mathrm{G}}$ » proporciona resultados semejantes a los de Charlier, al ser dependiente de los mismos factores mencionados (Tablas 1 y 2).

Taylor (1961) ideó un índice de agregación «b» que consistía en la regresión de la varianza sobre la media. Posee la ventaja de que puede ser ilustrado mediante una recta de regresión. Además, al operar con los valores obtenidos en cada uno de los muestreos realizados durante el período de estudio, tiene en cuenta las fluctuaciones del modelo espacial. En las tablas 1 y 2 , se observa como algunas especies (Scaurus punctatus, Rhytidoderes plicatus) que presentan valores medios de contagio para los índices anteriormente calculados a partir de las capturas totales, con la ley de potencia de Taylor expresan algunos de los niveles más elevados de agregación. Este hecho es debido a que estas especies poseen fuertes niveles de agregación en determinados períodos de su ciclo de actividad, por lo que el índice de Taylor, al tener en cuenta los diferentes períodos de muestreo, detecta esta situación, mientras que los índices anteriores no son capaces de apreciarla, al operar con la captura total.

Un parámetro muy utilizado como índice de agregación es «K» de la Distribución Binomial Negativa. Este índice está influenciado por el tamaño de la muestra y de la unidad de muestreo (Cole, 1946; Morris, 1954). Sin embargo, manteniendo constantes estos factores, proporciona una media muy útil del nivel de agregación de una población, siendo a la vez muy sensible al tipo de hábitat y al estado de desarrollo de las poblaciones (Hairston, 1959; Waters, 1954). Por otra parte, sus valores son inversos a los del índice de Morisita «I $\delta$ » (Morisita, 1959), que es el índice global más eficaz, al no depender del tamaño de la captura ni de la densidad media (Elliot, 1977). 
Lloyd (1967) ideó un índice de agregación « $\stackrel{*}{X}$ ( («mean crowding») muy adecuado para animales móviles, pero como puede apreciarse en las tablas 1 y 2 , se encuentra muy influenciado por la densidad (Southwood, 1978). Esta limitación puede corregirse dividiendo el índice por la media « $\overline{\mathrm{X}} / \overline{\mathrm{X}}$ « («patchiness») (Lloyd, 1967). Los resultados que se obtienen son casi idénticos a los obtenidos por el índice de Morisita (Tablas 1 y 2).

Iwao (1968) e Iwao y Kuno (1968) operando de la misma manera que Taylor, definen un nuevo índice de agregación « $\beta$ » («density contagiousness coefficient»), obtenido a partir de una regresión de la «mean crowding» $(\mathrm{X})$ sobre las medias $(\overline{\mathrm{X}})$ estimadas en varios muestreos consecutivos. Los diferentes valores de « $\beta$ » se expresan en las tablas 1 y 2 , y se representan las regresiones de algunas poblaciones, a modo comparativo, en las figuras 3 y 4 . Como puede apreciarse, este índice es el que mejor detecta las disposiciones espaciales encontradas, ya que posee las ventajas del de la Ley de Potencia de Taylor y además, fluctúa en intervalos mayores, por lo que es más fácil ajustar un nivel definitivo de agregación a cada población.

Para facilitar la interpretación de los valores de los índices calculados con los datos de las capturas totales, se presentan distintas gráficas de distribución de las capturas de 38 poblaciones en las tramas de trampas en el pastizal y pinar (Figuras 5 y 6 ). Como puede apreciarse, algunas poblaciones, que coinciden por lo general con las dominantes (de los Santos, 1983), como Pterostichus globosus ebenus, Pimelia costata, Tentyria platyceps, Pterostichus crenulatus, Bembidion varium, Thylacites latithorax, se encuentran muy extendidas por el pastizal; otras poblaciones se localizan en zonas muy recluidas, como ocurre con Orthomus sp. y Anysorhinchus sp. Existen, por otra parte, especies limitadas a zonas muy características, como Grabrasida parallela y Rhytirhinus $s p$. y por último, otras que sólo se recolectan en un reducido número de trampas, como Brachinus sclopeta, Platynus dorsalis o Licinus punctatulus granulatus.

Al objeto de detectar cambios estacionales en la disposición espacial de las poblaciones estudiadas, se calculó el índice de Morisita, por las ventajas anteriormente expuestas-, para cada una de las muestras temporales obtenidas durante el período de estudio. Las figuras 7 y 8 representan las variaciones temporales de este índice para 15 pobla- 

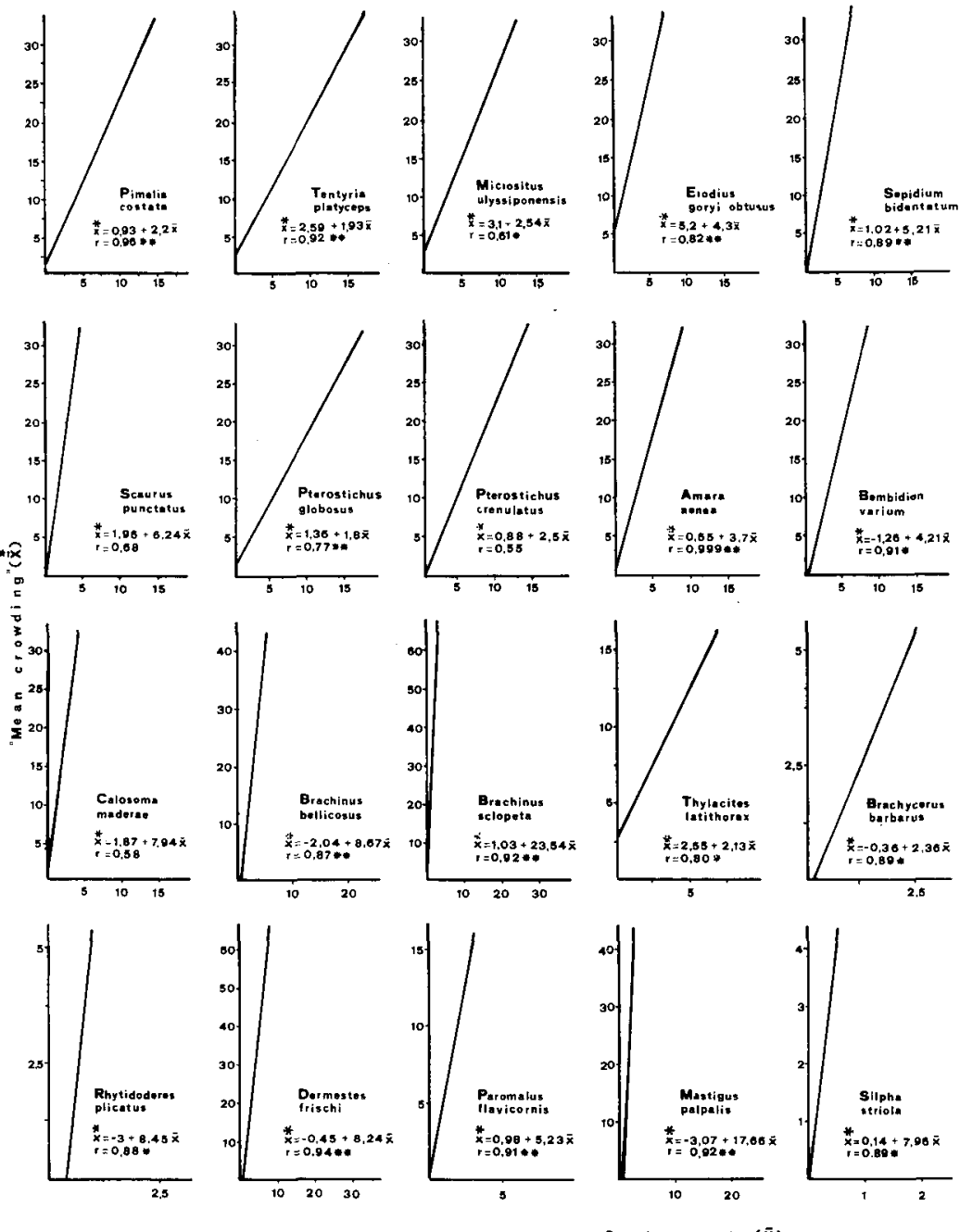

Densidad media $(\bar{x})$

Figura 3. - Relación entre la «mean crowding» (X) y la densidad media (X) (Indice de Iwao) de una serie de muestras para 20 especies de coleópteros del pastizal. $* \mathrm{P}<0,05 \quad * * \mathrm{P}<0,01$ 


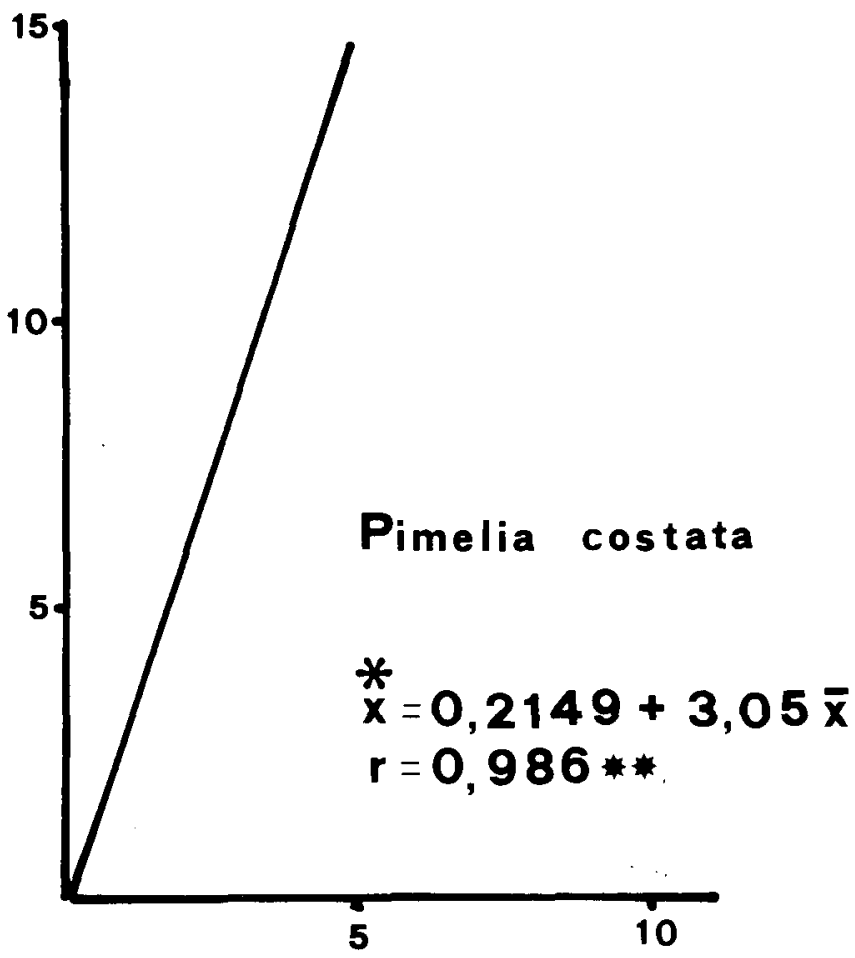

Figura 4. -- Relación entre la «mean crowding» (X) y la densidad media (X) (Indice de Iwao) de una serie de muestras para 2 especies de coleópteros del pinar. $\quad * \mathrm{P}<0.05 * * * \mathrm{P}<0.01$

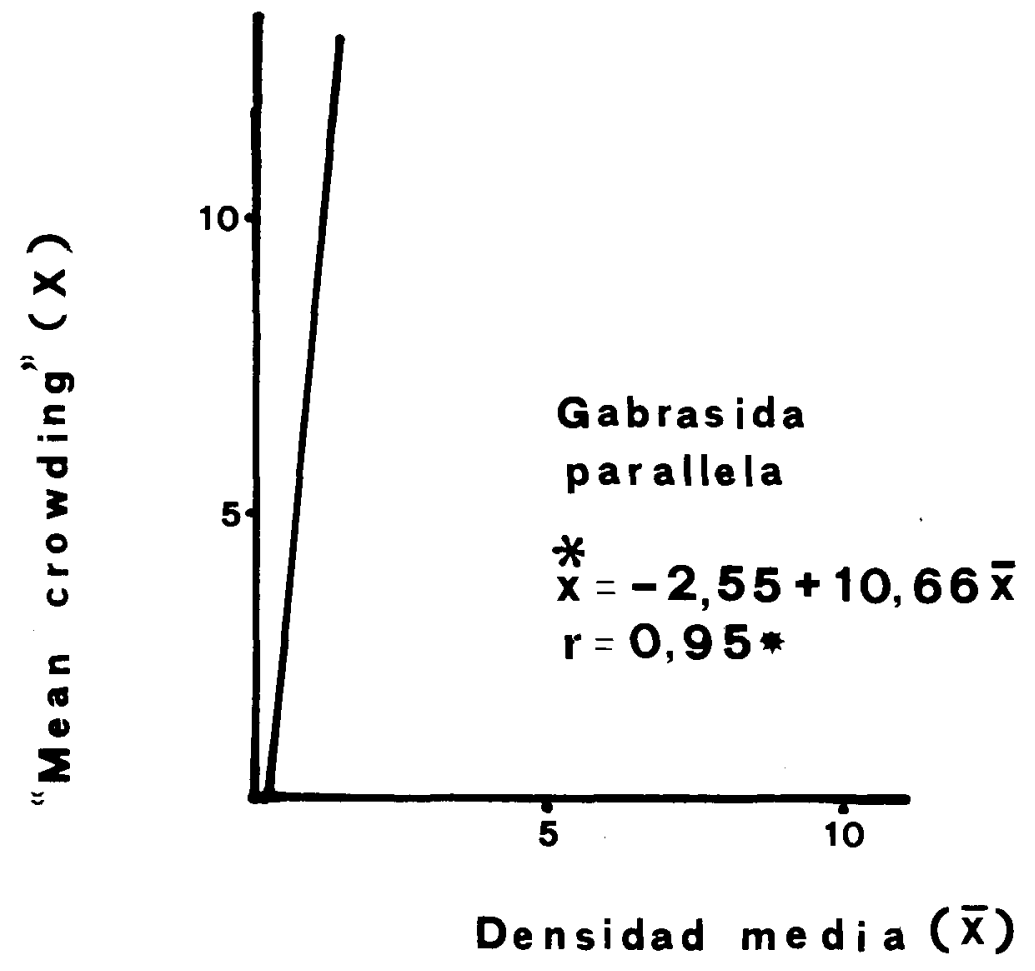


Pterostichus globosus

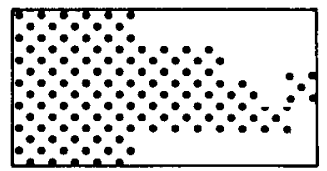

Piorostichus cronulatus

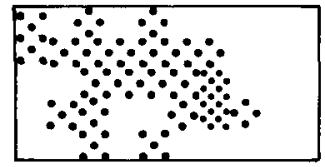

Platynus dorsalis

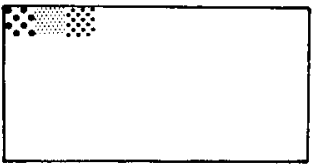

Pimulia costata

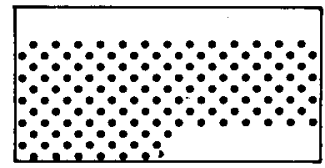

Sepidium bidentatum

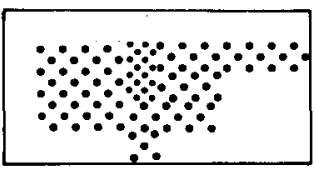

Thylacites latithorax
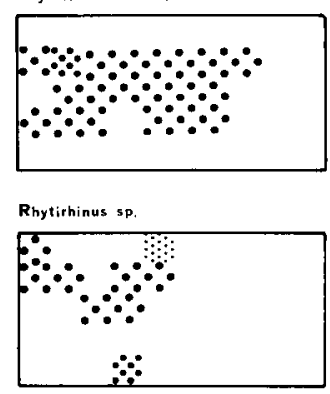

Paromalus flavicornis

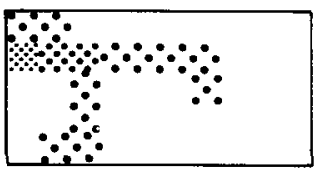

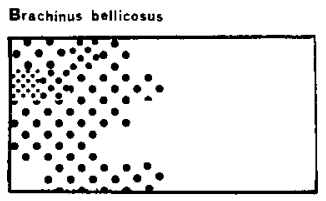

Calosoma maderae

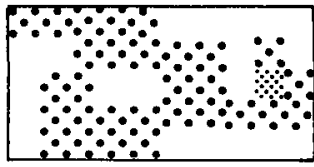

Madrocarabus lucitanicus

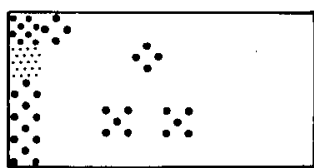

Tentyria platyceps

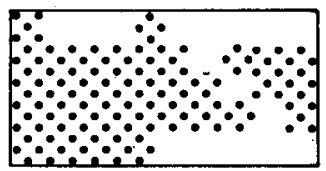

Scaurus punctatus

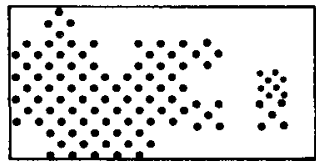

Brachycerus barbarus

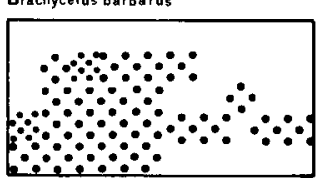

Anysorhynchus sp.

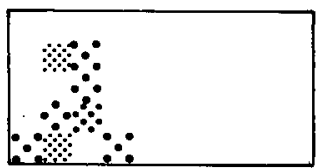

Moloe prosicarabaeus

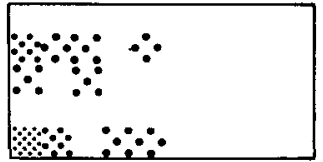

Brachinus selopets

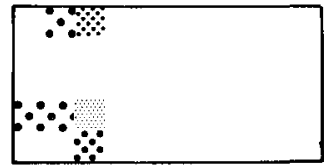

Bembidian varium

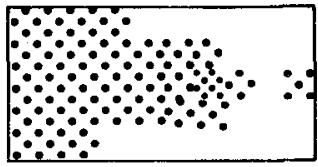

Licinus puctatulus
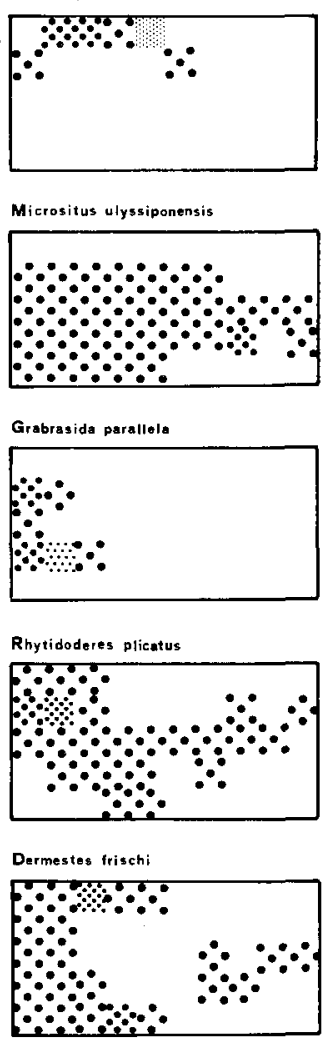

Trox perlatus

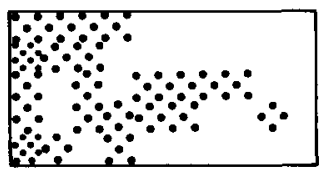

Amsra senea

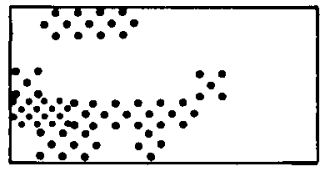

Orthomus sp.

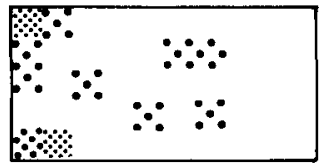

Silphs striols

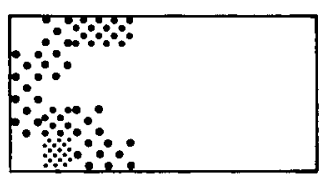

Erodius goryi obtusus

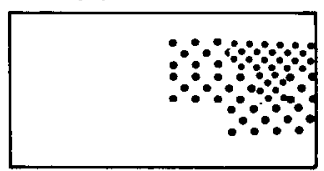

Litoborus planicollis

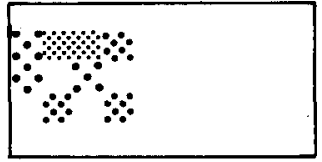

Brachycerus callosus

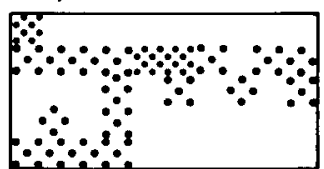

Mastigus palpelis

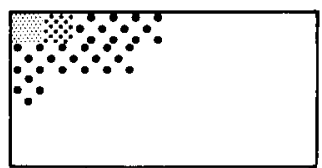

Thyphoeus momus

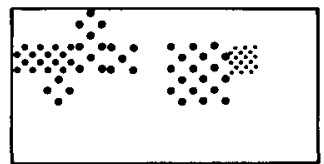


ciones de coleópteros del pastizal y 2 del pinar, consideradas como las más importantes para la comunidad en cuanto a sus valores de frecuencia y dominancia.

Como se puede apreciar, existen importantes fluctuaciones en el nivel de agregación para cada una de las poblaciones consideradas. De una forma general se pueden apreciar en los resultados determinados patrones de distribución temporal, entre los que destacan:

a) Especies que comienzan su actividad con débil nivel de contagio y que posteriormente, a lo largo de su período de actividad, se incrementa, volviendo a disminuir al final del mismo (Pterostichus crenulatus, Calosoma maderae, Brachycerus barbarus, Scaurus punctatus, Mastigus palpalis).

b) Especies que comienzan su actividad con valores bajos de agregación y que posteriormente se incrementa gradualmente a lo largo de su período de actividad (Pterostichus globosus).

c) Especies que comienzan su actividad con fuertes niveles de agregación y que posteriormente disminuye gradualmente (Pimelia costata, Micrositus ulyssiponensis, Thylacites latithorax).

d) Especies con valores altos de agregación al principio y al final de su período de actividad Tentyria platyceps, Erodius goryi obtusus, Paromalus flavicornis). Dentro de este último tipo, se pueden incluir especies que presentan un patrón estacional más complejo, como es el caso de Brachinus bellicosus, cuyas poblaciones sufren una dispersión durante las últimas dos semanas de su período de actividad, o Sepidium bidentatum y Dermestes frischi con fuertes fluctuaciones a lo largo de su período de actividad.

Salvo Mastigus palpalis $(\mathrm{r}=+0,74 \mathrm{P}<0,05)$ las poblaciones de coleópteros estudiadas no presentan correlaciones estadísticamente significativas entre los índices de agregación espacial y las densidades en actividad de las poblaciones, aunque en algunos casos los valores sean relativamente altos.

Este hecho puede justificarse, para algunas poblaciones, por la presencia de un cambio temporal en sus comportamientos gregarios. Así, 
Brachinus bellicosus, como se vio anteriormente, durante la primera mitad de su ciclo de actividad no se dispersa y presenta una correlación positiva ( $\mathrm{r}=+0,56 \mathrm{P}>0,05)$, mientras que, en su segunda mitad, los individuos llevan a cabo una marcada dispersión por el pastizal, habiéndose detectado una correlación significativa de signo negativo $(\mathrm{r}=$ $0,75 \mathrm{P}<0,05$ ). Al calcular el coeficiente de correlación para la totalidad de su período de actividad, el valor disminuye drásticamente $(r=+0,02)$. Esto apoya la necesidad de tener en cuenta, en los estudios de modelos espaciales, la variación estacional de las poblaciones.

De una manera global, las especies consideradas como dominantes presentan los niveles más bajos de agregación; las acompañantes poseen niveles intermedios, mientras que las accesorias se pueden considerar como gregarias, graduación que puede comprobarse, en este estudio, dentro de la familia Tenebrionidae (Figura 3).

\section{DISCUSIÓN.}

Los coleópteros terrestres de superficie, al igual que la mayoría de los organismos vivos, se encuentran distribuidos de una forma generalmente contagiosa (Grum, 1973, 1978). El tamaño e intensidad de los «agregados» vendrá determinado por la distribución de los factores ambientales del medio donde se desarrollan las poblaciones.

Las diferencias en el nivel de agregación, detectadas en las poblaciones estudiadas, se pueden explicar de una forma general por necesidades microclimáticas, capacidad de dispersión, tamaño de las poblaciones y distintos estímulos de agregación.

Thiele (1969) demuestra como las especies de la familia Carabidae, que se reproducen en Otoño, son en su mayoría nocturnas y típicas de hábitats forestales húmedos; mientras que las especies que se reproducen en Primavera son indiferentes a la luz y típicas de zonas abiertas de escasa humedad. En este estudio, las especies que se reproducen en Otoño (de los Santos, 1983) como Licinus punctatulus granulatus, Platynus dorsalis, Orthomus sp., poseen los niveles de agregación más elevados (Tabla 1), estando limitadas a zonas muy determinadas del pastizal (sector con influencia de estratos arboreos y de matorral higrofíticos). (Figura 5). 
Las especies que se reproducen en Primavera (Amara aenea, Bembidion varium, Calosoma maderae, Pterostichus crenulatus) presentan, por el contrario, niveles mucho más débiles de agregación, localizándose en las zonas más abiertas (Figura 5).

Las especies que poseen amplios períodos de actividad (8-9 meses) presentan niveles de agregación que se ajustan a sus diferentes fases reproductoras. De una forma general, los estados de vitelogénesis coinciden con los niveles máximos de agregación. Así, Pterostichus globosus ebenus presenta su contagio máximo al final de la primavera, período cuyas condiciones climáticas determinan la maduración de sus gonadas (Krehan, 1970; Hürka, 1975).

Los tenebriónidos como Pimelia costata, Tentyria platyceps y Micrositus ulyssiponensis, tienen su período de vitelogénesis al comienzo de la Primavera, después de su período de invernación, presentándose, por tanto, en esta estación, los niveles más elevados de agregación (Figura 7 y 8).

El caso más peculiar es el de Brachinus bellicosus, especie típicamente gregaria que, junto con Brachinus sclopeta, posee uno de los niveles de agregación global más elevado (Tabla 1). Según refleja la figura 7, Brachinus bellicosus, durante el período de vitelogénesis (comienzo del invierno) se dan niveles de agregación muy altos. Posteriormente, en su fase de actividad sexual, se produce una fuerte dispersión de la población, volviéndose a agrupar una vez finalizado el período de cópulas. A continuación vuelve a dispersarse, interpretándose como una búsqueda de condiciones más favorables para realizar la puesta. Un modelo de distribución similar al descrito, ha sido desarrollado por Wautier (1967, 1971 a y b) en experimentos de laboratorio con el género Brachinus. Los individuos de este género utilizan sus explosiones secretoras, además de mecanismos de defensa, como estimulante para la agregación (Dean, 1979).

Así pues, la intensidad de los agregados está ligada, en muchas especies, a determinadas modificaciones fisiológicas relacionadas con la reproducción. Un hecho muy conocido es el caso de las termitas, donde se ha demostrado un antagonismo entre sexualidad y sociabilidad (Verron, 1963).

Por otro lado, las especies de pequeño tamaño como Mastigus pal- 


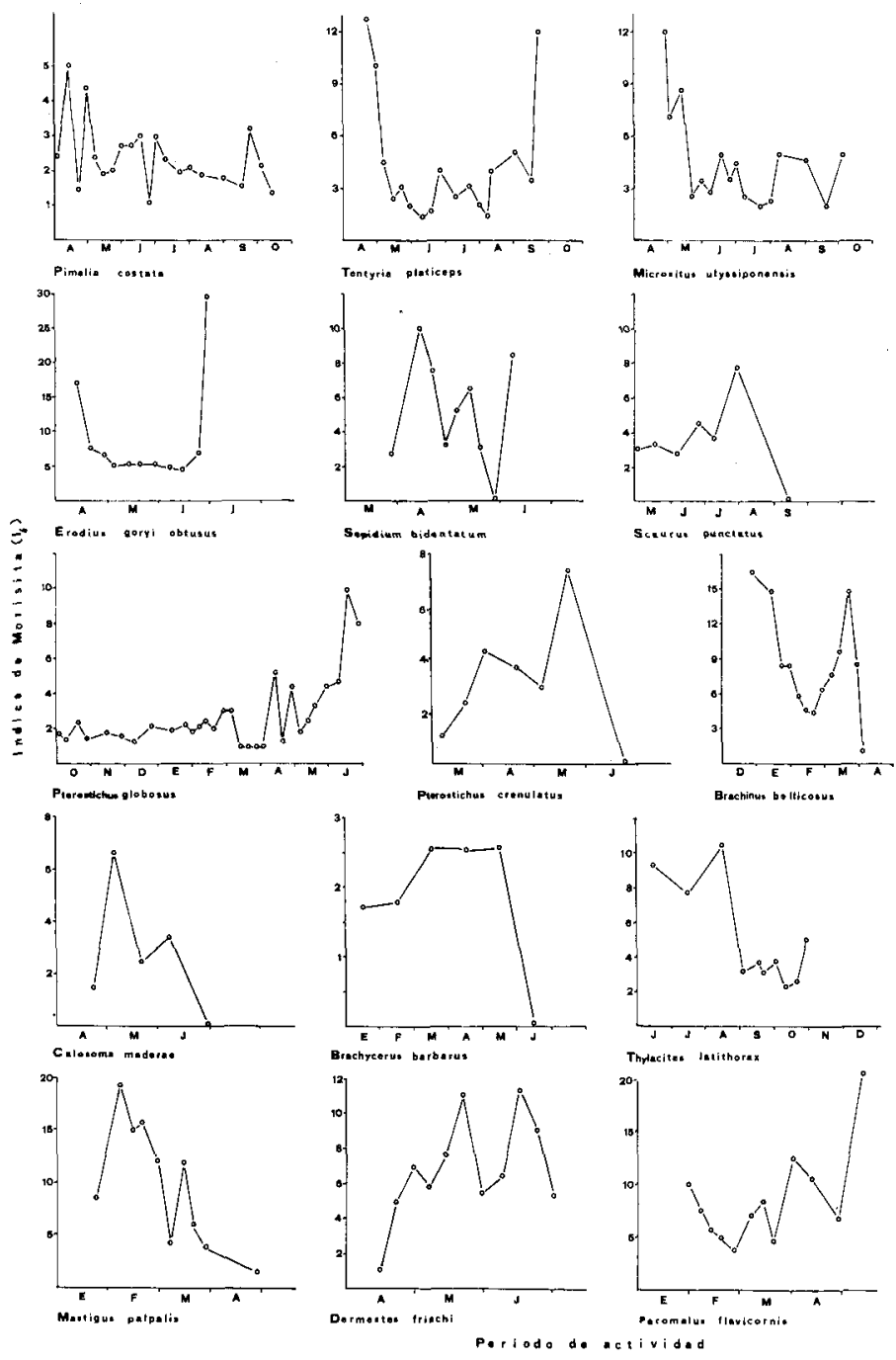

Figura 7. - Valores del lndice de Morisita $\left(I_{\delta}\right)$ para cada una de las muestras obtenidas a lo largo del período de actividad de 15 poblaciones de coleópteros en el pastizal 


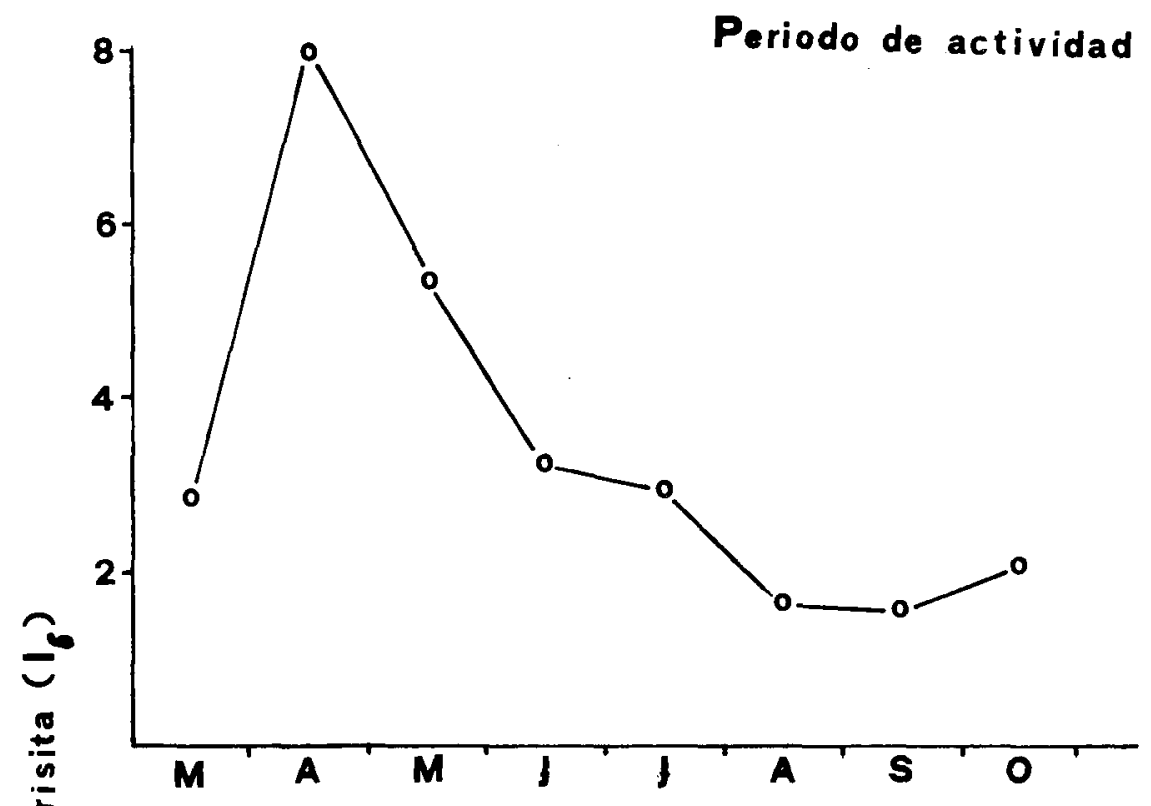

Pimelia costata

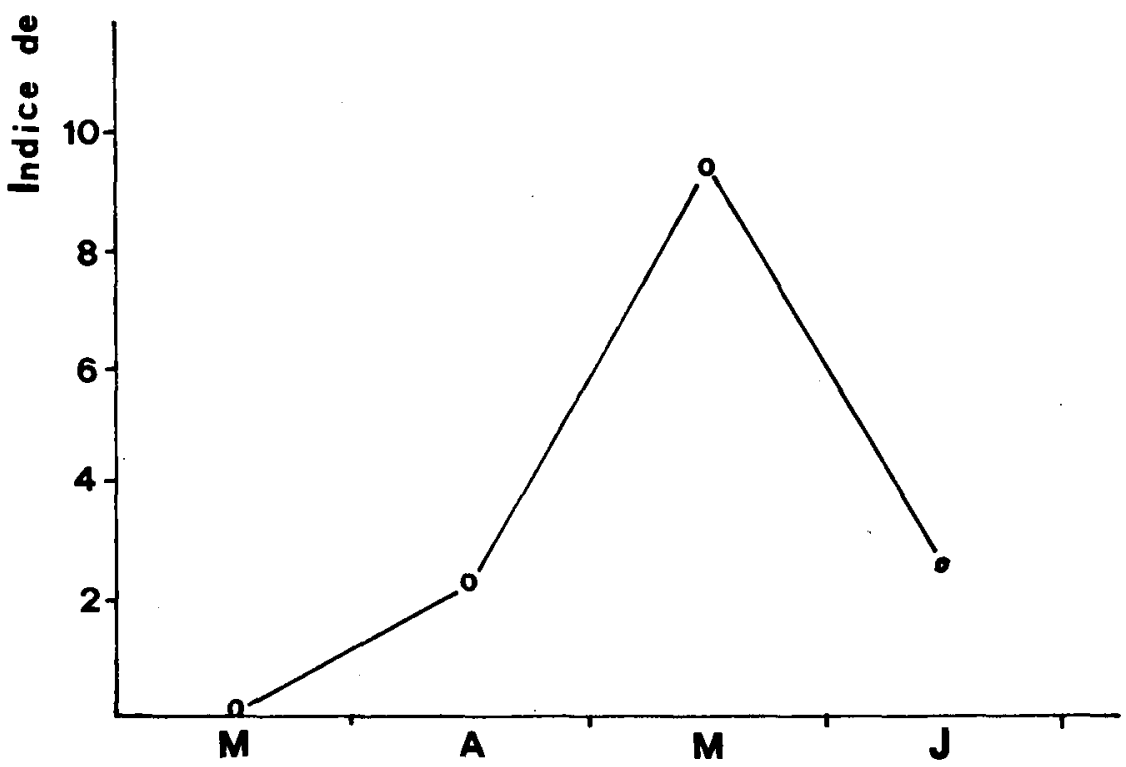

Grabrasida parallela

Figura 8. - Valores del Indice de Morisita $\left(\mathrm{I}_{\delta}\right)$ para cada una de las muestras obtenidas a lo largo del período de actividades de 2 poblaciones de coleópteros en el pinar 
palis (Fam. Scydmaenidae), Paromalus flavicornis (Fam. Histeridae) y Dermestes frischi (Fam. Dermestidae), presentan niveles de agregación muy elevados, siendo consideradas como buenos indicadores biológicos de los estados de conservación de los hábitats (Kleiners, 1977). Estos valores altos (Figura 5) no son de extrañar si tenemos en cuenta que el tamaño de los individuos de una especie parece ser el factor más importante, mediante el cual se puede estimar la amplitud del nicho (McArthur y Levins, 1967).

Lavigne (1978) ha demostrado como las especies de Calosoma y Microlestes se congregan allí donde existe una alta concentración de presas. Los grupos predadores al igual que los fitófagos deben tener sus picos de actividad acoplados a su fuente alimenticia. En este estudio, estos grupos no presentan valores globales de agregación muy elevados (Tabla 1). La distribución estacional se presenta en picos acoplados a los de las curvas de densidad en actividad (de los Santos, 1983). Estas poblaciones comienzan su actividad adoptando un modelo espacial más o menos disperso, y posteriormente van agrupándose en zonas determinadas donde existe una gran concentración de presas, detectándose en las gráficas una subida gradual de los valores de agregación (Pterostichus crenulatus, Calosoma maderae, Brachycerus barbarus -fitófago-) según se aprecia en la Figura 7. Al agotarse las presas la población se dispersaría de nuevo, traduciéndose en un descenso gradual de los índices de agregación.

Independientemente de las consideraciones anteriormente expuestas, la disposición espacial de las poblaciones depende, en último término del tamaño y distribución espacial de los grupos e individuos dentro de ellos (Elliot, 1977).

Andrewartha y Birch (1954) discuten las desventajas de las poblaciones con baja densidad en relación con el riesgo de no encontrar parejas y de que algún componente del medio pueda serles desfavorable. Una respuesta a este riesgo puede ser la tendencia a disponerse espacialmente de una forma contagiosa. Den Boer (1979) define a las poblaciones de coleópteros de superficie como «grupos interactuantes». Los grupos ocuparían un espacio equivalente a las distancias medias que cubren los individuos de la población durante su período de actividad, desde sus centros de dispersión. Estos grupos estarían interrelacionados por individuos migrantes. 
Si integramos la zona de estudio dentro de una gran área geográfica, sobre la cual existen diferentes ambientes con similares características microclimáticas, los grupos que componen una población se pueden encontrar aislados, debido fundamentalmente a las actividades agrícolas, o como grupos interrelacionados por ecotonos o interfases, que gradualmente unirían unos con otros. Dentro de cada grupo existen individuos migrantes (Baars, 1979), capaces de realizar marchas unidireccionales cubriendo grandes distancias. Van Huitzen (1977) y Jonhson (1969) han demostrado que estas migraciones ocurren justamente unas semanas antes del período de reproducción, lo que aseguraría un intercambio genético entre los diferentes grupos que componen una población.

En el caso de las poblaciones aisladas, el peligro de extinción es muy alto, mientras que en el segundo caso, el riesgo de extinción está amortiguado por la heterogeneidad ambiental (Reddingius, 1971). El aislamiento de los hábitats naturales por actividades agrícolas provoca una pérdida continua del poder de dispersión (Den Boer, 1980) de las poblaciones, ya que los individuos que se dispersan tienen una probabilidad menor de sobrevivir que los individuos que quedan en la población, por lo que las características genéticas ligadas a la dispersión (individuos migrantes) entran en un proceso de feed-back positivo, que generalmente conducen a la eliminación de la población.

Por último, para compensar este proceso de aislamiento y extinción de las poblaciones, se podrían construir sendas seminaturales junto a los caminos y carreteras (Elton, 1927). Una densa franja cubierta por piedras podría facilitar la dispersión de muchas especies entre restos de áreas naturales, poniendo en contacto poblaciones con modelos espaciales fuertemente contagiosos.

\section{AGRADECIMIENTOS.}

Deseamos expresar nuestro agradecimiento a Fernando Sancho y Pilar Martín por sus comentarios críticos y estímulos durante el desarrollo de todo el estudio. A los Doctores, D. Francisco Español, D. Miguel Angel Alonso y D. Bussi Corrado por su colaboración en la determinación del material. Asimismo, este estudio ha sido llevado a cabo, gracias a la inestimable ayuda de compañeros y amigos, a los que, de igual modo, queremos manifestarles nuestro agradecimiento: Enrique Bernal y Francisco Osuna por su ayuda en la recolección y ordenación 
del material de campo; Francisco Sánchez y José de los Santos por facilitarnos, desinteresadamente, sus vehículos para los desplazamientos al campo.

\section{BIBLIOGRAFÍA}

ADIS J., 1979. Problems of interpreting arthropod sampling with pitfall traps. Zool. Anz., 202 (3-4): 177-184.

ANDREWARTHA H. G.; BIRCH L. C., 1954. The distribution and abundance of animals. Univ. Chicago Press, 783 págs.

BAARS M. A., 1979. Catches in pit-fall traps in relation to mean densities of carabid beetles. Oecologia (Berlín), 41 (1): 25-46.

COLE L. C., 1946. A theory for analyzing contagiously distributed populations. Ecology, 27: 329-341.

DEAN J., 1979. Defensive reaction time of bombardier beetles. An investigation of the speed of a chemical defense, J. Chem. Ecol., 5 (5): 691-701.

DEBAUCHE H. R., 1958. Problemes de biocénotique. Rev. Quest. Sci., 129: $58-59$.

DEN BOER P. J. 1979. The significance of dispersal power for the survival of species, with special references to the carabid beetles in a cultivated countryside. Fortschr. Zool., 25 (2-3): 79-94. 
DEN BOER P. J., 1980. Exclusion or coexistence and the taxonomic or ecological relationship between species. Neth. J. Zool., 30 (2): 278-306.

DUFFEY E., 1962. A population study of spiders in limestone grassland. Description of study area. Sampling methods and population characteristics. J. Animal Ecology, 31: 571-599.

ELLIOT J. M., 1977. Some methods for the statistical analysis of samples of benthic invertebrates (2nd ed.). Fresh. Biol. Assoc. Sci., Pub. No. 25, 156 págs.

ELTON C. S., 1927. Animal Ecology. London. Sidwick and Jackson.

GIST C. S.; GROSSLEY D. A., 1973. A method for quantitying pitfall trapping. Environ. Ent. 2: 951-952.

GONZÁLEZ BERNÁLDEZ F., 1978. Síntesis de los ecosistemas del Bajo Guadalquivir. En: García Novo y otros: Doñana. Prospección e inventario de ecosistemas: 9-21. ICONA. Monografía No. 18. 244 págs.

GREEN R. H., 1966. Measurement of non-randomness in spatial distributions. Researches Popul. Ecol. Kyoto Univ. 8: (1): 1-7.

GREENSLADE P. J. M., 1964. Pit-fall trapping as a method for studying populations of Carabidae (Col.). J. Anim. Ecol., 33: 301-310.

GREIG-SMITH P., 1964. Quantitative plant ecology (2nd ed.). Butterwoths, London, 256 págs.

GRUM L., 1973. Patterns of Carabus arcensis Hbst. distribution within different habitats. Bull. Acad. pol. Sci. Cl. II, Sér. Sci. Biol., 21: 229-233.

GRUM L., 1978. Mechanisms governing rate and direction of energy flow through carabid populations. Pol. Ecol. Stud., 4 (2): 129-175.

GRUM L., 1980. Methods of aproximate estimation of energy flow through carabid populations. Ekol. Polska, 28 (1): 129-149. 
HAIRSTON N. G., 1959. Species abundance and organisation. Eco$\log y, 40: 404-416$.

HAYES W. B., 1970. the occuracy of pit-fall trapping for the sand-beach isopod Tylos punctatus. Ecology, 51 (3): 514-516.

HURKA K., 1975. Laboratory studies on the life cycle of Pterostichus melanarius (Illig.) (Col. Carabidae). Vest. Cs. Spol. Zool., 39: 265-274.

IWAO S., 1968. A new regression method for analyzing the agregation pattern of animal populations. Res. Pol. Ecol., 10: 1-20.

IWAO S.; KUNO E., 1968. Use of the regression of mean crowding on mean density for estimating sample size and the transformation of data for the analysis of variance. Researches Popul. Ecol. Kyoto Univ., 10: 210-214.

JOHNSON C. G., 1969. Migration and dispersal of insects by flight. Methuen, London.

JOOSSE E. N. G., 1975. Pitfall-trapping as a method for studying surface dwelling Collembola. Z. Morph. Oekol. Tiere, 55: 587-596.

KREHAN I., 1970. The control of annual periodicity and diapause in overwinring larvae and adults of genus Pterostichus (Col. Carabidae). Oecologia (Berlín), 6: 58-105.

KLEINERT J., 1977. Soil surface coleoptera of northeastern Slovakia with regard on family Carabidae. Biologia (Bratislava) 32: 307-315.

LAVIGNE R., 1978. The effect for resource enrichment on the abundance of individual carabid species (Col.). Cordulia, 4 (3): 100-107.

LEFKOVITCH L. P., 1966. An index of spatial distribution. Researches Popul. Ecol. Kyoto Univ., 8: 89-92.

LEVECHE CH., 1972. Mollusques benthiques du Lac Tchad: ecologie, etude des peuplements et estimation des biomasses. Cah. O.R.S.T.O.M., ser Hidrobiol., 6 (1): 3-45. 
LLOYD M., 1967. Mean crowding. J. Anim. Ecol., 36: 1-30.

LUFF M., 1975. Some features influencing the efficiency of pitfall-traps. Oecologia (Berlín), 19: 345-357.

LUFF M., 1979. The biology of the ground beetle Harpalus rufipes in a strawberry field in Northumberland. Ann. Appl. Biol., 94: 153-164.

MCARTHUR R. H.; LEVINS R., 1967. The limiting similarity, convergence and divergence of coexisting species. Amer. Natur., 101: 377-385.

MITCHELL B., 1963. Ecology of two carabid beetles, Bembidion lampros Herbst. and Trechus quadristriatus Schrank. I. Life cycles and feeding behaviour. J. Anim. Ecol., 32: 289-299.

MORISITA M., 1959. Measuring the dispersion of individuals and analysis of the distributional patterns. Mem. Fac. Sci. Kyushu Univ. Ser. E. Biol., 2: 215-235.

MORRIS R. F., 1954. A sequential sampling technique for spruce bud worm egg surveys. Can. J. Zool., 32: 302-313.

OBRTEL R., 1971. Soil-surface coleoptera in a lowland forest. Acta Sci. Nat. Acad. Sci. Bohemoslov. Brno., 5 (7): 1-47.

PRICE J.; SHEPARD M., 1980. Sampling ground predators in soydean fields. Sampling Methods in soybean entomology. SpringerVerlag, New York. 587 págs.

REDDINGIUS J., 1971. Gambling for existence. A discussion of some theoretical problems in animal population ecology. Acta Biotheor. (Leiden), 20: 1-208.

ROJAS B. A., 1964. La Binomial Negativa y la estimación de intensidad de plagas en el suelo. Fitolecnia Latinamer. 1 (1): 27-36.

SANTOS A. de los; MONTES C.; RAMÍREZ-DÍAZ L., 1983. Un nuevo diseño de trampa de caída para el estudio de poblaciones de coleópteros terrestres de superficie. Mediterránea 6 
SANTOS A. de los, 1983. Biología y ecología de algunas poblaciones de coleópteros terrestres en dos ecosistemas del Bajo Guadalquivir (S. W. España). Tesis Doctoral. Universidad de Murcia.

SOUTHWOOD T. R. E., 1978. Ecological Methods, with particular reference to the study insect populations (2nd ed.). Chapman and Hall, 524 págs.

TAYLOR L. R., 1961. Aggregation, variance and the mean. Nature (Lond.), 189: 732-735.

THIELE H. U., 1969. Connections between diurnalism, annual periodicity and habitat-selection in carabid beetles (Col.). Oecologia (Berlín), 29: 27-49.

VAN HIUTZEN T. H. P., 1977. The significance of flight activity in the life cycle of Amara plebeja Gyll. (Col. Carabidae).

VERRON H., 1963. Role des stimuli chimiques dans l'attraction sociale chez Calotermes flavicollis Fabr. Insectes sociaux, 10: 167-335.

WATERS W. E., 1954. A quantitative measure of aggregation in insectes. J. econ. ent., 52: 1.180-1.184.

WAUTIER J.; WAUTIER V., 1967. Modification en repport avec l'activité sexualle du comportament gregaire chez Brachinus sclopeta (Col. Carabidae). Ann. Soc. Entomol. de France, 3 (3); 1-8.

WAUTIER V., 1971 a. Comparaison du comportement grégaire des males et des femelles de Brachinus sclopeta a l'époque de la reproduction (Col. Carabidae). Bull. Soc. Entomol. France, 76: 7-9.

WAUTIER V., 1971 b. Un phenomene social chez les Coleopteres: le gregarisme des Brachinus. Insectes sociaux, 3: 1-84. 